\title{
Effect of selected sampling media, flow rate, and time on the sampling efficiency of a liquid impinger packed with glass beads for the collection of airborne viruses
}

\author{
Yen-Chi Chen $\cdot$ I-Jen Wang $\cdot$ Chih-Ching Cheng $\cdot$ Yu-Chiao Wu • \\ Chun-Hsuan Bai $\cdot$ Kuo-Pin Yu
}

Received: 8 July 2020/ Accepted: 11 December 2020/Published online: 13 January 2021

(C) The Author(s), under exclusive licence to Springer Nature B.V. part of Springer Nature 2021

\begin{abstract}
The liquid impingers can be used for sampling of viral aerosols, such as COVID-19 virus, influenza, and measles. However, the lowest cutoff diameter of commercially available liquid impingers was about $0.3 \mu \mathrm{m}$, and the physical collection efficiency for nano-bioaerosol is only about $10-20 \%$. Here, we enhanced the impinger's collection efficiency and recovery of viable viral aerosols by using packed glass beads and selected sampling media ( $1 \%$ peptone and lysogeny broth, LB). Single-stranded RNA (ssRNA) MS2 bacteriophage with uranine (as a physical tracer) was used as model viral aerosols. The effects of different sampling flow rates $(4,6$, and 12.5 L per minute) and different sampling time (10, 20, and $30 \mathrm{~min}$ ) on the collection efficiency and recovery of
\end{abstract}

Yen-Chi Chen and I-Jen Wang have contributed equally.

Y.-C. Chen - I.-J. Wang - C.-C. Cheng ·

Y.-C. Wu - C.-H. Bai - K.-P. Yu (ه)

Institute of Environmental and Occupational Health

Sciences, National Yang-Ming University, No. 155, Sec.

2, Li-Nong Street, Taipei City 11221, Taiwan (ROC)

e-mail: kpyu@ym.edu.tw; f89541105@gmail.com

\section{I.-J. Wang}

Department of Pediatrics, Taipei Hospital, Ministry of Health and Welfare, No.127, Su-Yuan Road,

New Taipei City 24213, Taiwan (ROC)

I.-J. Wang

School of Medicine, National Yang-Ming University,

Taipei, Taiwan
MS2 aerosols were also tested. Collection efficiency and recovery of viable viral aerosols were analyzed as a function of sampling media, flow rate, and sampling time and packed glass beads by using a general linear model. Although the packed glass beads considerably enhanced the collection efficiency of the liquid impinger for MS2 aerosols, the recovery of viable MS2 becomes lower due to the higher pressure drop across the impinger. Using peptone or LB as sampling media, reducing sampling flow rate, and decreasing sampling time was proven to improve the recovery of viable MS2. Conclusively, this study provides some practical methods to improve the collection efficiency of liquid impinger for viral aerosols and preserve their viability.

\author{
I.-J. Wang \\ College of Public Health, China Medical University, \\ Taichung, Taiwan \\ I.-J. Wang \\ National Institute of Environmental Health Sciences, \\ National Health Research Institutes, Miaoli, Taiwan \\ I.-J. Wang \\ National Taiwan University Hospital, National Taiwan \\ University, Taipei, Taiwan
}


Keywords Bioaerosols · Virus collection efficiency $\cdot$ Viability

\section{Introduction}

Viruses can become airborne and aerosolized in many different ways, e.g., human or animal physiological activities such as sneezing, coughing, or breathing. These viruses can spread in the air and potentially cause respiratory infections (Douwes et al. 2003). Airborne transmission of viruses, such as coronaviruses (He et al. 2004; Kim et al. 2016; Paules et al. 2020), influenza (Moser et al. 1979; Herfst et al. 2012), measles (Riley et al. 1978; Bloch et al. 1985; Remington et al. 1985), porcine epidemic diarrhea viruses (Alonso et al. 2014), and foot-and-mouth disease viruses (Mikkelsen et al. 2003), plays a critical role in epidemic infection. The probability of airborne viruses causing an infection depends on their particle size and their characteristics, such as infectious dose and survivability in the air. Some outbreak events seem to be related to ventilation and air-conditioner issues (Tomlinson and Cockram 2003), which can result in positive virus detection in the air of hospitals (Booth et al. 2005). Airborne viruses may survive and remain infectious for hours in aerosols (Bloch et al. 1985; van Doremalen et al. 2020; Ong et al. 2020), and appropriate air sampling methods are required to detect them in the air. There are three common air sampling methods: inertial impaction, filtration (Burge and Solomon 1987), and impingement (Hogan et al. 2005; Terzieva et al. 1996). Liquid impingers have a distinct advantage in the collection of viral particles because the biological agent in liquid media would not be dehydrated and is more convenient for post-analysis (Lin et al. 2000; Tseng and Li 2005).

The collection efficiency of impingers depends on the aerodynamic diameters of airborne particles, and the main sampling mechanisms include inertial impaction, diffusion, and centrifugation ( $\mathrm{Yu}$ et al. 2016; Tseng and Li 2005; Terzieva et al. 1996). However, the minimum cutoff size of particles was $300 \mathrm{~nm}$ for commercial impingers and showed very low collection efficiency (10-20\%) for particles smaller than $100 \mathrm{~nm}$ (Hogan et al. 2005). On the other hand, the virus aerosol transmission route has been particularly controversial because there is a lack of direct proof for viruses that can be transmitted through the air, which is partly due to the challenges of detecting airborne viruses (Tellier 2006). The diameter of airborne viral particles was $0.03-0.2 \mu \mathrm{m}$, which was smaller than the cutoff size of conventional bioaerosol samplers (Verreault et al. 2008). Therefore, enhancing the collection efficiency for airborne viral particles and their viability recovery is important for detecting pathogenic airborne virus particles.

Our previous studies have demonstrated that using a granular bed can enhance the performance of the allglass impinger 30 (AGI-30), with a very high collection efficiency (>98\%) for particles of $20-500 \mathrm{~nm}$ (Yu et al. 2016). However, many factors, such as relative humidity $(\mathrm{RH})$, temperature, re-aerosolization, sampling media, sampling time, and sampling flowrate, could affect the collection efficiency and recovery of viruses (Verreault et al. 2008). Several liquid media have been used for sampling viral aerosols, including filtered deionized water (DW) (Chang and Chou 2011; Liu et al. 2012), phosphatebuffered saline (PBS) (Liu et al. 2012), and Tween 80 mixture (deionized water with $1 \%$ peptone, $0.01 \%$ Tween 80 and $0.005 \%$ antifoam A)(Chang and Chou 2011). A surfactant type of media, such as Tween 80 , could reduce the surface tension of water and decrease the impact on the viruses. Moreover, polyhydroxy compounds (Liu et al. 2012; Schaffer et al. 1976; Benbough 1969) and peptone (Hemmes et al. 1960) liquids can stabilize viruses, maintain their activity, and increase the detectability of airborne viruses. Therefore, choosing a suitable liquid media is one of the key factors for preserving the activity of the viruses during sampling (Alvarez et al. 1995; Shahamat et al. 1997).

Herein, three selected media, $1 \%$ peptone, lysogeny broth (LB), and disinfected water, were used as the sampling liquids to assess their effects on the collection efficiency for viral aerosols and the recovery of the viable virus from sampling liquid. Furthermore, this study systematically investigated the effects of sampler flow rates and sample times on the collection efficiency for viral aerosols and their recovery. In the experiments, single-stranded RNA (ssRNA) MS2 bacteriophage with uranine (a physical tracer) (Zuo et al. 2014) was used as the model viral aerosols. This study aims to figure out the optimal conditions for the sampling of airborne virus particles. 


\section{Experimental methods}

\subsection{Preparation of suspension of model microorganisms}

The Bioresource Collection and Research Center (BCRC) in Taiwan provided the model microorganisms, MS2 bacteriophage (BCRC70235) and its host Escherichia coli (BCRC50354), for the generation of viral aerosols. MS2 bacteriophages have singlestranded RNA genomes, and their particles have an approximate diameter of 25-27 nm. MS2 bacteriophages are often used as a surrogate for pathogenic viruses (e.g., poliovirus, rhinovirus, FMD virus, and norovirus) (Jain et al. 2006; Bae and Schwab 2008; Verreault et al. 2008). For the preparation of MS2 suspension, first, the viable MS2 bacteriophages infected the $E$. coli host and then multiplicated on the double-layer culture plates [tryptic soy agar (TSA) (Difco, Becton, Dickinson \& Company, USA)]. Next, the MS2 culture plates were frozen at $-20{ }^{\circ} \mathrm{C}$ for $5 \mathrm{~h}$. After that, the MS2 culture plates were thawed at room temperature and produced liquid containing MS2. This liquid was centrifuged at 3,000 rpm for $10 \mathrm{~min}$ and then passed through a $0.22-\mu \mathrm{m}$ filter to remove the $E$. coli host residues. The resultant liquid (titer of MS2 $=2 \pm 0.6 \times 10^{16}$ plaque-forming units $/ \mathrm{mL}$ ) was used for the generation of MS2 aerosols.

\subsection{Experimental setup}

The experimental setup is schematically shown in Fig. 1. The sampler used in the experiments was an AGI-30 (ACE GLASS Inc.; Vineland, NJ, USA). To enhance its collection efficiency for viral particles, we packed the AGI-30 sampler with glass beads ( $3 \mathrm{~mm}$ diameter), as shown in Fig. 1a (Yu et al. 2016). The manufacturer of these 3-mm glass beads (borosilicate glass) is Boeckel Co. (GmbH Co.) (BOECO Germany). According to $\mathrm{Yu}$ et al. (2016), packing with 3 - $\mathrm{mm}$ glass beads with a depth of $10 \mathrm{~cm}$ is the optimal condition for AGI-30 sampler to enhance its collection efficiency for airborne particles. Before each experiment, the piping line of the whole system was disinfected with $75 \%$ alcohol. Previous studies have shown $70 \%$ ethanol can reduce MS2 titer by $0.7 \log 10$ in $2 \mathrm{~min}$ and $3.68 \log 10$ in $20 \mathrm{~min}$ (Jones et al. 1991; Maillard et al. 1994). After evaporation, there is no $75 \%$ alcohol residue, and thus it is better than other disinfectants, such as sodium hypochlorite (bleach), for viral decontamination.

The supply air for the viral aerosol generation was provided by a zero-air system, which contained an air compressor to deliver high-pressure air, a highefficiency particulate air (HEPA) filter to remove airborne bioaerosol particles, and a diffusion dryer to eliminate moisture. Then this supply air was introduced to a three-jet Collison nebulizer (BGI Inc.; Waltham, MA, USA), containing MS2 bacteriophage

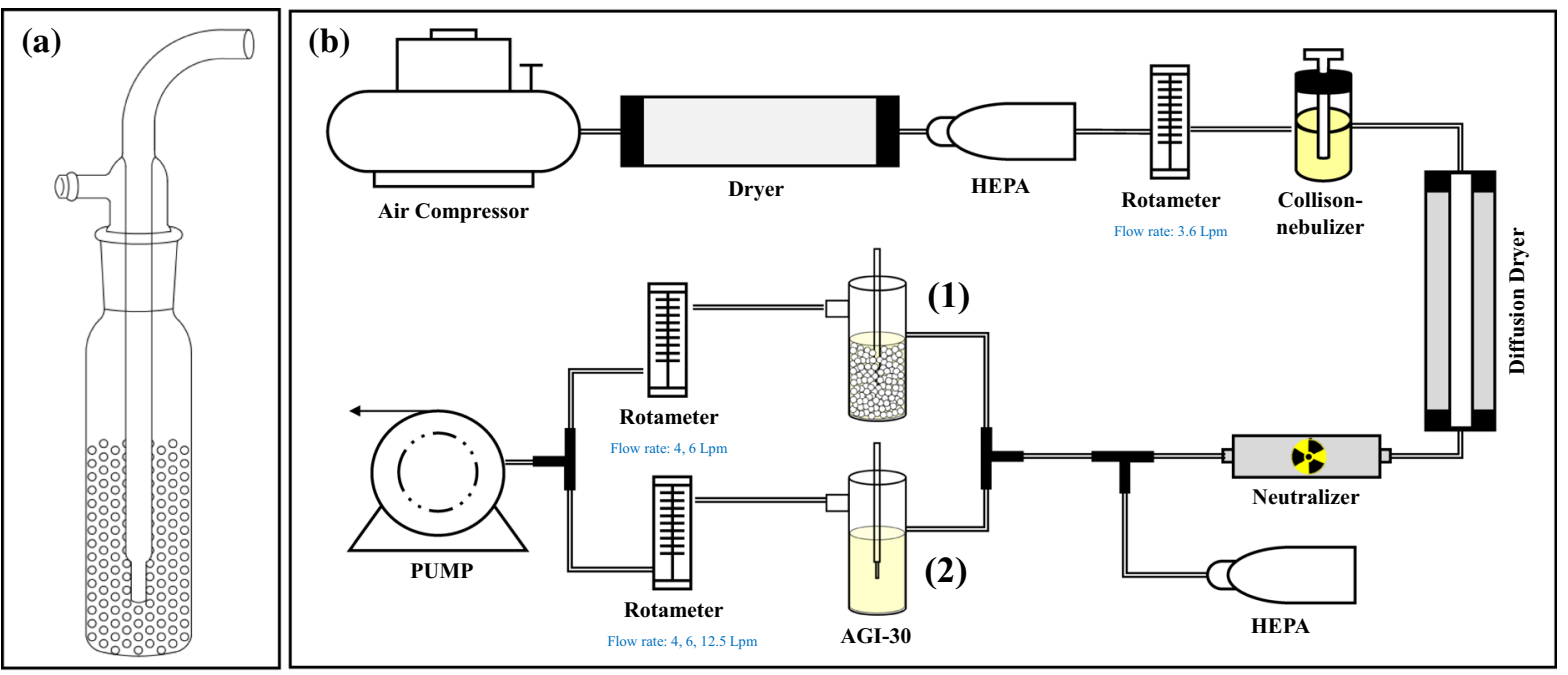

Fig. 1 a AGI30 with packed glass beads and b schematic diagram of the experimental setup 
suspension $(35 \mathrm{~mL})$ with $0.15 \%$ of uranine (as a fluorescent particle tracer, fluorescein sodium, Sigma Chemical Co; St. Louis, MO, USA) (Zuo et al. 2014), at a flow rate of 3.6 (liter per minute, $\mathrm{Lpm}$ ) to generate MS2 droplets. After that, these droplets passed through a homemade diffusion dryer $(60 \mathrm{~cm}$ long and $1 \mathrm{~cm}$ in diameter) to form droplet nuclei and a $\mathrm{Kr}-$ 85 radioactive aerosol neutralizer (Model 3077, TSI Inc., USA) to become neutrally charged.

Then, the air steam containing MS2 aerosols was sampled by (1) the AGI-30 packed with glass beads at a flow rate of 4 and $6 \mathrm{Lpm}$ or (2) the AGI-30 without packing at a flow rate of 4,6 , and $12.5 \mathrm{Lpm}$, simultaneously. The concentration of the fluorescent particle tracers and titer of viable MS2 bacteriophages was determined by multimode microplate readers (485 and $515 \mathrm{~nm}$, Infinite 200; Tecan, Grödig, Austria) and enumerated by the plaque-forming unit (PFU) in the double-layered cultures, respectively. We investigated the effects of several parameters on the collection efficiency of the AGI-30 packed with glass beads, including the sampling flow rate, sampling liquid, and sampling time. Table 1 summarizes the parameters used in the experiments. Each experiment was performed in triplicate. The number concentrations and size distributions of the virus aerosol particles were measured before the AGI-30 sampler by using a scanning mobility particle sizer (SMPS) (model 3936L76; TSI, Inc.; Shoreview, MN, USA).

\subsection{Determination of sampling efficiency}

To evaluate the loss of aerosols in the piping system (including diffusion dryer and neutralizer) and physical collection efficiency of AGI-30 for the viral particle at various sampling conditions, we compared the tracer concentration in the nebulizer and sampler and calculated the fluorescent tracer (uranine) collection efficiency by the following equation:

$E_{\text {tracer }}=\frac{C_{\text {tracer,s }} \times V_{\mathrm{s}}}{Q_{\text {ne }} \times C_{\text {tracer,ne }} \times t} \times 100 \%$

where $C_{\text {tracer,s }}$ is the concentration of the tracer collected by the sampler at sampling time $t ; V_{s}(\mathrm{~mL})$ represents the volume of the sampled liquid; $Q_{\mathrm{ne}}(\mathrm{mL}$ $\mathrm{min}^{-1}$ ) is the liquid nebulization flow rate of the nebulizer; $C_{\text {tracer,ne }}$ is the concentration of the tracer in the nebulizer; and $t(\mathrm{~min})$ represents the sampling time.

In addition to physical collection efficiency, we also evaluated the biological collection efficiency of the sampler for viable MS2. The following equation was used to evaluate the biological collection efficiency of the sampler for viable MS2 bacteriophages $\left(E_{\mathrm{PFU}}\right)$ :

$E_{\mathrm{PFU}}=\frac{C_{\mathrm{PFU}, \mathrm{s}} \times V_{\mathrm{s}}}{Q_{\mathrm{ne}} \times C_{\mathrm{PFU}, \mathrm{ne}} \times t} \times 100 \%$

where $C_{\mathrm{PFU}, \mathrm{s}}$ represents the concentrations of viable MS2 bacteriophages collected in the sampler; $C_{\mathrm{PFU} \text {, ne }}$ is the concentrations of viable MS2 bacteriophages in the nebulizer. The titer of viable MS2 bacteriophages was determined by counting the PFUs in the doublelayered cultures.

Table 1 Summary of parameter values used in the experiments

\begin{tabular}{ll}
\hline Parameters & Condition \\
\hline Test particles & Bacteriophages MS2 \\
Tracer & $0.15 \%$ Uranine (Fluorescein sodium salt) \\
Diameter of test particles $(\mathrm{nm})$ & $30-300$ \\
Glass beads diameter $(\mathrm{mm})$ & 3 \\
The glass beads packed depth $(\mathrm{cm})$ & 10 \\
Liquid in sampler & Disinfected (Dis) water, 1\% Peptone, 0.15\% Luria-Bertani (LB Broth) \\
Volume of sampling liquid in the impinger (mL) & 33 \\
Sampling flow rate through impinger (liter per min, lpm) & with packing: 4, 6 non-packing: 4, 6, 12.5 \\
Sampling time (min) & $10,20,30$ \\
\hline
\end{tabular}




\subsection{Data analysis}

The data are based on the arithmetic means of the triplicate analyses. Collection efficiency and recovery of uranine and viable viral aerosols were analyzed as a function of sampling liquids, packed glass beads, sampling flow rate, and sampling time by using a general linear model (GLM). All the significance levels were set to $\alpha=0.05$, and the analyses were conducted using SPSS for Windows, version 20.0 (IBM Corporation; Armonk, New York, USA). The GLM is a suitable framework for comparing how several variables (sampling liquids, with or without packed glass beads, sampling flow rate, and sampling time) influence different continuous variables (Collection efficiency and recovery of uranine and viable viral aerosols, and sampler quality factor).

\section{Results and discussion}

3.1 Effect of packed glass beads and sampling flow rates on the collection efficiency of AGI30 sampler

The particle size distribution of the generated MS2 bacteriophage aerosols is shown in Fig. 2. The geometric mean $(\mathrm{GM})$ was $98.2 \pm 1.72 \mathrm{~nm}$, and the



Fig. 2 Size distributions of submicrometre and ultrafine particles produced from the atomization of MS2 phage with $0.15 \%$ uranine. The arithmetic and geometric means are $150.1 \pm 2.14$ and $98.22 \pm 1.72 \mathrm{~nm}$, respectively. The presented size distributions indicate the mean by distributions of three repeats. The coefficient of variation between each repeat was less than $5 \%$ number concentration was $4 \times 10^{4}$ (particles $/ \mathrm{cm}^{3}$ ), which was comparable to the results in our previous study (MS2 bacteriophages with LB media GM = $107.5 \pm 1.83 \mathrm{~nm})(\mathrm{Yu}$ et al. 2016). Factors that can affect the particle size distribution include the media of viral suspension, concentration of virus, and flow rate of nebulizer (Yu et al. 2016; Hogan et al. 2005).

Figure 3 demonstrates the comparison between the tracer and MS2 bacteriophage (PFU) collection efficiency of the AGI-30 with packed glass beads and the AGI-30 without packing. Accordingly, the tracer collection efficiency of the AGI-30 samplers without and with packing ranged from $1.20 \pm 0.46$ to $14.09 \pm 3.41 \%$ and from $1.91 \pm 1.27$ to $44.49 \pm 15.86 \%$, respectively. While the PFU collection efficiency of the AGI-30 sampler without and with packing ranged from $0.62 \pm 0.06$ to $6.41 \pm 1.04 \%$ and from $0.59 \pm 0.05$ to $11.57 \pm 3.55 \%$, respectively. Generally, the AGI-30 with packing performed better than that without packing, and the enhancement effect of the packed glass beads on the collection efficiency was statistically significant, as shown in Table 2. Besides, increasing the sampling flow rate has a positive effect on collection efficiency, which was also statistically significant. The mechanism in the larger airborne particles, which contained more uranine molecules, and MS2 bacteriophages were mainly collected by inertial impaction, which worked better at a higher flow rate. Thus, raising the sampling flow rate would enhance the collection efficiency. A similar result has been reported by Hogan et al. (2005), where MS2 and T3 bacteriophages were used as experimental aerosol particles (Hogan et al. 2005). However, there was a critical pressure-drop limitation, and the sampling flow rate at 12.5 LPM was not applicable for the AGI30 packed with glass beads. A tracer collection efficiency of $44.5 \pm 15.8 \%$ was attained by the AGI-30 with packing and disinfected water (sampling liquid) and operating at a sampling flow rate of $6 \mathrm{Lpm}$. Under the same conditions, the AGI-30 without packing had a collection efficiency of only $10.7 \pm 2.8 \%$. Hogan et al. used three different liquid samplers (AGI-30, SKC BioSampler, and a frit bubbler), and their collection efficiency did not exceed $25 \%$ with a sampling flow rate between 3.5 and 12.5 LPM (Hogan et al. 2005). Packing the glass beads can enhance the collection efficiency of AGI-30 by either diffusion or impaction. Even at a lower sampling flow 

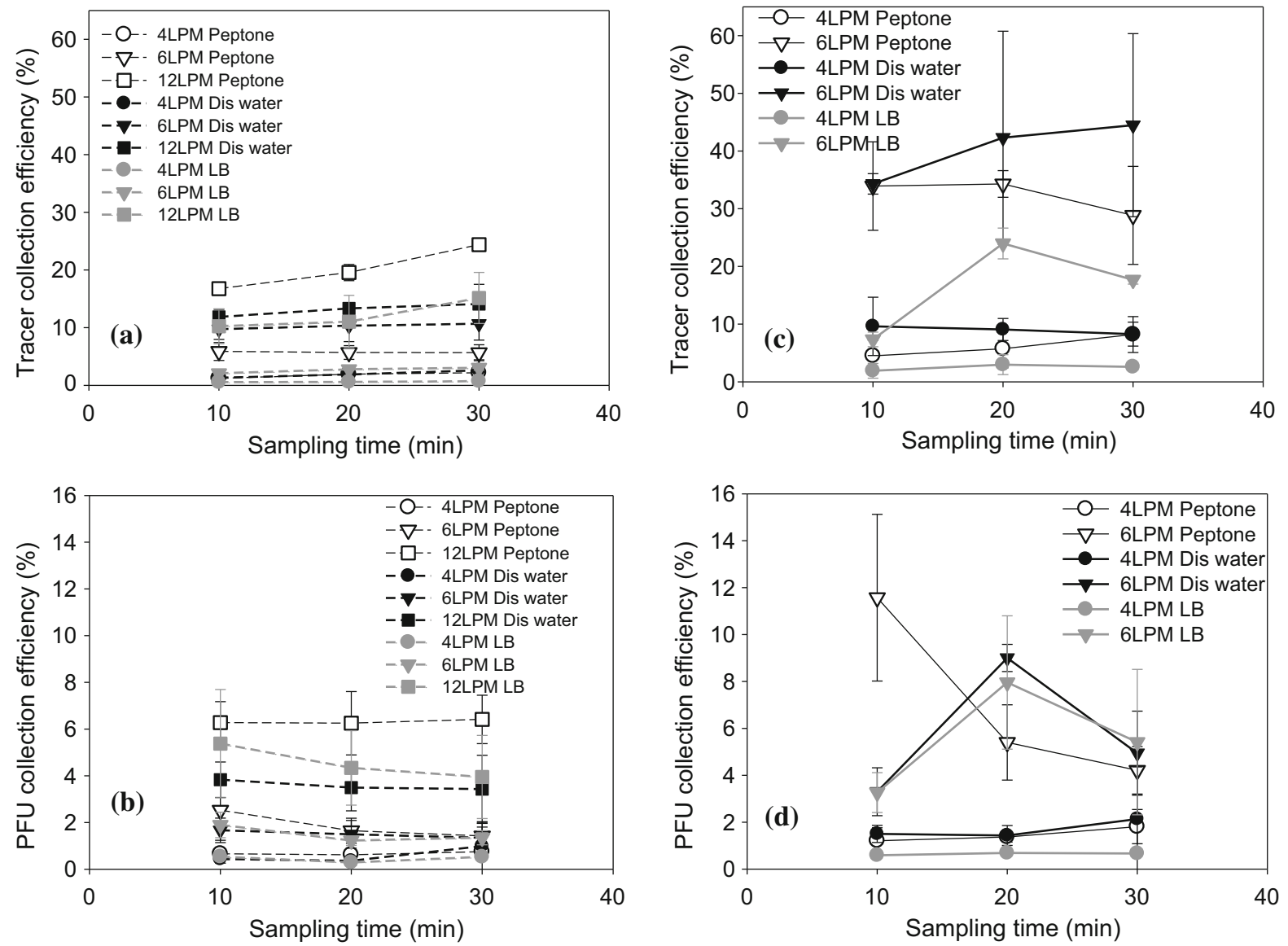

Fig. 3 The collection efficiency of the AGI-30 for airborne particles of a uranine b MS2 bacteriophage, and AGI-30 with packed glass beads for $\mathbf{c}$ uranine $\mathbf{d}$ MS2 bacteriophage

rate, the AGI-30 with packing retained a high collection efficiency for MS2 aerosol particles.

\subsection{Recovery of PFU and sampler quality factor}

Figure 3 also exhibits the comparison between the tracer and biological collection efficiency for MS2 bacteriophage aerosols, and the tracer collection efficiency was higher than the PFU collection efficiency. In this study, the fluorescent tracer collection efficiency $\left(E_{\text {tracer }}\right)$ imitated the maximum collection efficiency of the sampler for the viral aerosol particles in our experimental system, and $E_{\text {tracer }}$ also reflected the fraction of the nebulized viral aerosol arrival the sampler after penetrating through the diffusion dry, neutralizer, and piping line. Therefore, the ratio of $E_{\mathrm{PFU}}$ to $E_{\text {tracer }}$ expressed as the following equation could be applied to evaluate the recovery of viable MS2 bacteriophages from the samplers $\left(R_{\mathrm{PFU}}\right)$ :

$R_{\mathrm{PFU}}=\frac{E_{\mathrm{PFU}}}{E_{\text {tracer }}} \times 100 \%$

As demonstrated in Fig. 4, the recovery of PFU $\left(R_{\mathrm{PFU}}\right)$ ranged from $9.6 \pm 3.0 \%$ to $91.1 \pm 26.3 \%$. The optimal condition for the recovery of PFU is using lysogeny broth (LB) as the sampling liquid and operating at a lower sampling flowrate at 4 LPM. According to the analysis result of the General Linear Model, reducing sampling time to less than $10 \mathrm{~min}$ and using a $1 \%$ peptone solution or LB as the sampling liquids were significantly beneficial to maintain the viability of the collected MS2 bacteriophages. Both peptone and LB media contain a substantial amount of peptide, which could protect viral structures and functions and keep the virus from unfavorable 


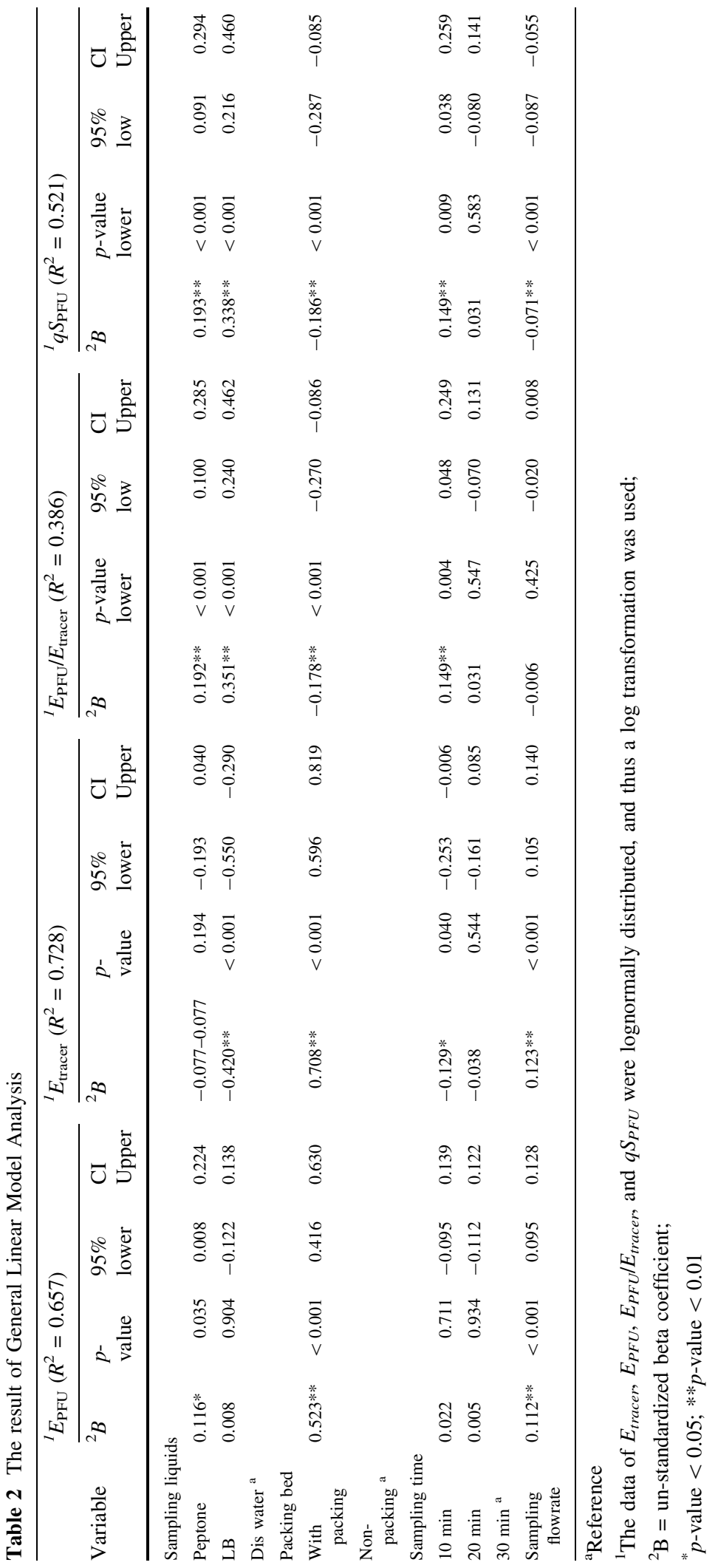




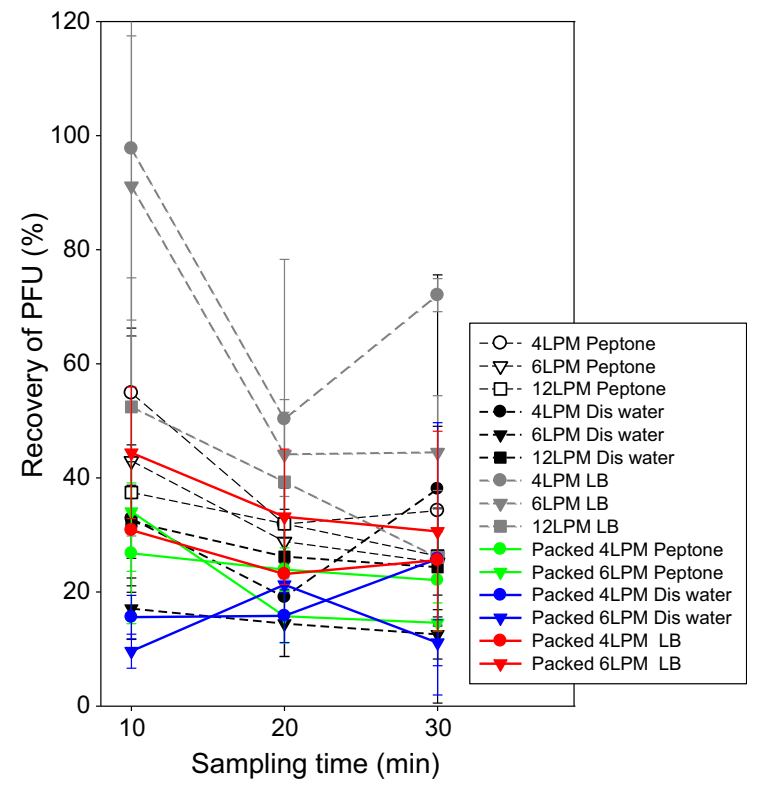

Fig. 4 The recovery of viable MS2 bacteriophage collection by the AGI-30 without and with packed glass beads

conditions. A review article by Verreault et al. has mentioned that adding polyhydroxy compounds and peptones to the spray fluid is protective for the recovery of airborne viruses by sampling (Verreault et al. 2008). Liu et al. (2012) also indicated that wetting agents reduce the surface tension of water and lower the impact on bacteriophages in air samples (Liu et al. 2012). Besides, reducing sampling time could reduce the re-aerosolization of collected MS2 bacteriophages from the sampler (Riemenschneider et al. 2010) and decrease the time exposed to unfavorable conditions of vigorous bubbling at room temperature (Verreault et al. 2008), and thus favorable to the recovery of PFU.

However, the packed glass beads in the AGI-30 sampler had a negative effect on the recovery of PFU, as shown in Table 2. This result might be relevant to the substantial increase of sampler's pressure drop during sampling due to the packed glass beads. Increasing pressure drop would put stress on the collected viruses, which may result in the loss of viral viability. An ideal sampler requires both a higher collection efficiency and recovery for viral aerosols and lower pressure drop, which can also reduce the stress on the sampled microbes and decrease the loading of the sampling pump. Herein, we used the "sampler quality factor" as a criterion to evaluate the

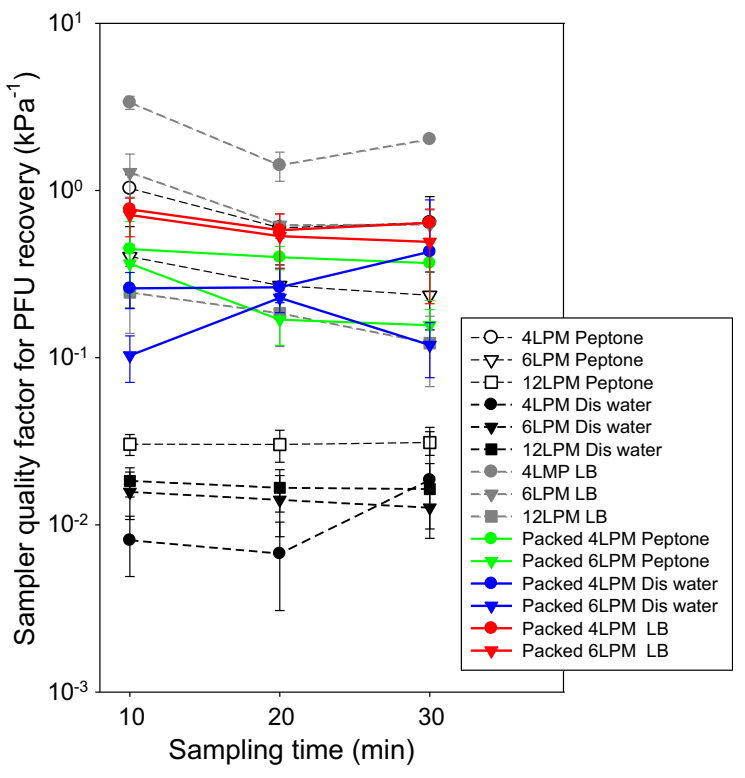

Fig. 5 The sampler quality factor of AGI-30 sampler without and with packed glass beads for MS2 bacteriophage recovery

samplers, which is expressed as the following equation:

$q S=\left(E_{\mathrm{PFU}} / E_{\text {tracer }}\right) / \Delta p$

where $q S$ is the sampler quality factor; $\Delta p(\mathrm{~mm}-\mathrm{Hg})$ represents the pressure drop through the sampler. As demonstrated in Fig. 5, the $q S$ of AGI-30 with and without packed glass beads for the recovery of viable MS2 bacteriophage ranged from $0.1031 \pm 0.0319$ to $0.7714 \pm 0.0225 \mathrm{kPa}^{-1}$ and from $0.0067 \pm 0.0037$ to $1.0293 \pm 0.2133 \mathrm{kPa}^{-1}$, respectively. According to Table 2, the decrease of the sampling flow rate had a positive effect on the $q S$ for the collection of viable MS2 bacteriophage. However, the packed glass beads can still statistically significantly reduce the $q S$ for the collection of viable MS2 bacteriophage, as shown in Table 2. Both the packing of glass beads and increasing sampling flow rate would raise the pressure drop across the sampler. However, the effect of pressure drop on the recovery of PFU has been adjusted in the $q S$ calculation, and thus factors other than pressure drop may affect the recovery of MS2 bacteriophages. The packed glass beads might increase the re-aerosolization and decrease the mixing of the sampling media, which might lead to a lower recovery of PFU. 


\subsection{Limitation and future work}

In fact, the feasibility of using this optimized condition for a real-world sampling is still unknown since the concentration of airborne viruses in the air is much far lower than the experimental condition and the size and durability of the viruses are varied. Therefore, this is a limitation of this study and more experiments for the real-world sampling should be conducted to verify the finding obtained in the lab.

\section{Conclusions}

This study investigated the effectiveness of using packed glass beads to improve the collection efficiency of the liquid impinger for submicron viral aerosols. Moreover, the effects of sampling flow rate, sampling media, and sampling time on the collection efficiency and recovery of viable viral aerosols were systematically investigated. Although the packed glass beads can considerably enhance the collection efficiency of the liquid impinger, the recovery of the viable virus becomes statistically significantly lower due to the stresses, including higher pressure drop, introduced by the packing. In this study, three approaches have been proven to improve the recovery of viable viruses $\left(R_{\mathrm{PEFU}=\mathrm{P} / \mathrm{EFUtracer}}\right)$ and sampler quality factor $\left(q S_{\mathrm{PFU}}\right)$, including using peptone or LB media as sampling liquid, reducing sampling flow rate, and decreasing sampling time. Conclusively, this study provides optimal settings of sampling liquids, flow rates, and times for liquid impinger packed with glass beads for submicron viral aerosol sampling.

Acknowledgments This work was supported by the Ministry of Science and Technology of Taiwan (MOST 105-2221-E-010002-MY3) and Taipei Hospital, Ministry of Health and Welfare of Taiwan (10602).

\section{References}

Alonso, C., Goede, D. P., Morrison, R. B., Davies, P. R., Rovira, A., Marthaler, D. G., et al. (2014). Evidence of infectivity of airborne porcine epidemic diarrhea virus and detection of airborne viral RNA at long distances from infected herds. Veterinary Research, 45(1), 73.

Alvarez, A. J., Buttner, M. P., \& Stetzenbach, L. D. (1995). PCR for bioaerosol monitoring: sensitivity and environmental interference. Applied and Environment Microbiology, 61(10), 3639-3644.
Bae, J., \& Schwab, K. J. (2008). Evaluation of murine norovirus, feline calicivirus, poliovirus, and MS2 as surrogates for human norovirus in a model of viral persistence in surface water and groundwater. Applied and Environment Microbiology, 74(2), 477-484. https://doi.org/10.1128/AEM. 02095-06.

Benbough, J. (1969). The effect of relative humidity on the survival of airborne Semliki Forest virus. Journal of General Virology, 4(4), 473-477.

Bloch, A. B., Orenstein, W. A., Ewing, W. M., Spain, W. H., Mallison, G. F., Herrmann, K. L., et al. (1985). Measles outbreak in a pediatric practice: airborne transmission in an office setting. Pediatrics, 75(4), 676-683.

Booth, T. F., Kournikakis, B., Bastien, N., Ho, J., Kobasa, D., Stadnyk, L., et al. (2005). Detection of airborne severe acute respiratory syndrome (SARS) coronavirus and environmental contamination in SARS outbreak units. Journal of Infectious Diseases, 191(9), 1472-1477.

Burge, H. A., \& Solomon, W. R. (1987). Sampling and analysis of biological aerosols. Atmospheric Environment, 21(2), $451-456$.

Chang, C. W., \& Chou, F. C. (2011). Methodologies for quantifying culturable, viable, and total Legionella pneumophila in indoor air. Indoor Air, 21(4), 291-299. https:// doi.org/10.1111/j.1600-0668.2010.00701.x.

Douwes, J., Thorne, P., Pearce, N., \& Heederik, D. (2003). Bioaerosol health effects and exposure assessment: progress and prospects. The Annals of Occupational Hygiene, 47(3), 187-200.

He, L.-Y., Hu, M., Huang, X.-F., Yu, B.-D., Zhang, Y.-H., \& Liu, D.-Q. (2004). Measurement of emissions of fine particulate organic matter from Chinese cooking. Atmospheric Environment, 38(38), 6557-6564.

Hemmes, J., Winkler, K., \& Kool, S. (1960). Virus survival as a seasonal factor in influenza and poliomyelitis. Nature, 188(4748), 430-431.

Herfst, S., Schrauwen, E. J., Linster, M., Chutinimitkul, S., de Wit, E., Munster, V. J., et al. (2012). Airborne transmission of influenza $\mathrm{A} / \mathrm{H} 5 \mathrm{~N} 1$ virus between ferrets. Science, 336(6088), 1534-1541.

Hogan, C. J., Jr., Kettleson, E. M., Lee, M. H., Ramaswami, B., Angenent, L. T., \& Biswas, P. (2005). Sampling methodologies and dosage assessment techniques for submicrometre and ultrafine virus aerosol particles. Journal of Applied Microbiology, 99(6), 1422-1434. https://doi.org/ 10.1111/j.1365-2672.2005.02720.x.

Jain, R., Knorr, A. L., Bernacki, J., \& Srivastava, R. (2006). Investigation of bacteriophage MS2 viral dynamics using model discrimination analysis and the implications for phage therapy. Biotechnology Progress, 22(6), 1650-1658.

Jones, M., Bellamy, K., Alcock, R., \& Hudson, R. (1991). The use of bacteriophage MS2 as a model system to evaluate virucidal hand disinfectants. Journal of Hospital Infection, 17(4), 279-285.

Kim, S.-H., Chang, S. Y., Sung, M., Park, J. H., Bin Kim, H., Lee, H., et al. (2016). Extensive viable Middle East respiratory syndrome (MERS) coronavirus contamination in air and surrounding environment in MERS isolation wards. Reviews of Infectious Diseases, 63(3), 363-369.

Lin, X. J., Reponen, T., Willeke, K., Wang, Z., Grinshpun, S. A., \& Trunov, M. (2000). Survival of airborne microorganisms 
duringbswirling aerosol collection. Aerosol Sci Tech, 32, 184-196.

Liu, K., Wen, Z., Li, N., Yang, W., Wang, J., Hu, L., et al. (2012). Impact of relative humidity and collection media on mycobacteriophage D29 aerosol. Applied and Environment Microbiology, 78(5), 1466-1472.

Maillard, J.-Y., Beggs, T., Day, M., Hudson, R., \& Russell, A. (1994). Effect of biocides on MS2 and K coliphages. Applied and Environmental Microbiology, 60(6), 2205-2206.

Mikkelsen, T., Alexandersen, S., Astrup, P., Donaldson, A., Dunkerley, F., Gloster, J., et al. (2003). Investigation of airborne foot-and-mouth disease virus transmission during low-wind conditions in the early phase of the UK 2001 epidemic. Atmospheric Chemistry and Physics Discussions, 3(1), 677-703.

Moser, M. R., Bender, T. R., Margolis, H. S., Noble, G. R., Kendal, A. P., \& Ritter, D. G. (1979). An outbreak of influenza aboard a commercial airliner. American Journal of Epidemiology, 110(1), 1-6.

Ong, S. W. X., Tan, Y. K., Chia, P. Y., Lee, T. H., Ng, O. T., Wong, M. S. Y., et al. (2020). Air, Surface Environmental, and Personal Protective Equipment Contamination by Severe Acute Respiratory Syndrome Coronavirus 2 (SARS-CoV-2) From a Symptomatic Patient. The Journal of the American Medical Association. https://doi.org/10. 1001/jama.2020.3227.

Paules, C. I., Marston, H. D., \& Fauci, A. S. (2020). Coronavirus infections-more than just the common cold. The Journal of the American Medical Association, 323(8), 707-708.

Remington, P. L., Hall, W. N., Davis, I. H., Herald, A., \& Gunn, R. A. (1985). Airborne transmission of measles in a physician's office. The Journal of the American Medical Association, 253(11), 1574-1577.

Riemenschneider, L., Woo, M. H., Wu, C. Y., Lundgren, D., Wander, J., Lee, J. H., et al. (2010). Characterization of reaerosolization from impingers in an effort to improve airborne virus sampling. Journal of Applied Microbiology, 108(1), 315-324. https://doi.org/10.1111/j.1365-2672. 2009.04425.x.

Riley, E., Murphy, G., \& Riley, R. (1978). Airborne spread of measles in a suburban elementary school. American Journal of Epidemiology, 107(5), 421-432.
Schaffer, F., Soergel, M., \& Straube, D. (1976). Survival of airborne influenza virus: effects of propagating host, relative humidity, and composition of spray fluids. Archives of Virology, 51(4), 263-273.

Shahamat, M., Levin, M., Rahman, I., Grim, C., Heidelberg, J., Stelma, G., et al. (1997). Evaluation of media for recovery of aerosolized bacteria. Aerobiologia, 13(4), 219-226.

Tellier, R. (2006). Review of aerosol transmission of influenza A virus. Emerging Infectious Diseases, 12(11), 1657-1662. https://doi.org/10.3201/eid1211.060426.

Terzieva, S., Donnelly, J., Ulevicius, V., Grinshpun, S. A., Willeke, K., Stelma, G. N., et al. (1996). Comparison of methods for detection and enumeration of airborne microorganisms collected by liquid impingement. Applied and Environment Microbiology, 62(7), 2264-2272.

Tomlinson, B., \& Cockram, C. (2003). SARS: experience at Prince of Wales Hospital, Hong Kong. Lancet, 361, 1486-1487.

Tseng, C.-C., \& Li, C.-S. (2005). Collection efficiencies of aerosol samplers for virus-containing aerosols. Journal of Aerosol Science, 36(5-6), 593-607.

van Doremalen, N., Bushmaker, T., Morris, D. H., Holbrook, M. G., Gamble, A., Williamson, B. N., et al. (2020). Aerosol and Surface Stability of SARS-CoV-2 as Compared with SARS-CoV-1. New England Journal of Medicine. https:// doi.org/10.1056/NEJMc2004973.

Verreault, D., Moineau, S., \& Duchaine, C. (2008). Methods for sampling of airborne viruses. Microbiology and Molecular Biology Reviews, 72(3), 413-444. https://doi.org/10.1128/ MMBR.00002-08.

Yu, K. P., Chen, Y. P., Gong, J. Y., Chen, Y. C., \& Cheng, C. C. (2016). Improving the collection efficiency of the liquid impinger for ultrafine particles and viral aerosols by applying granular bed filtration. Journal of Aerosol Science, 101, 133-143.

Zuo, Z., Kuehn, T. H., Bekele, A. Z., Mor, S. K., Verma, H., Goyal, S. M., et al. (2014). Survival of airborne MS2 bacteriophage generated from human saliva, artificial saliva, and cell culture medium. Applied and Environment Microbiology, 80(9), 2796-2803. https://doi.org/10.1128/ AEM.00056-14. 